\title{
COMPORTAMIENTO DE LOS CULTIVARES DE PAPAYA SUNSET, SUNRISE Y DE LOS GENOTIPOS BAIXINHO DE SANTA AMALIA Y BH - 65 EN LA ZONA SUR DE LA ISLA DE TENERIFE ${ }^{1}$
}

\author{
MARIA CRISTINA RODRÍGUEZ PASTOR², MARÍA GLORIA LOBO RODRIGO³, \\ CARMEN LUISA SUÁREZ SÁNCHEZ ${ }^{4}$
}

RESUMEN-Los antecedentes de la papaya (Carica papaya L.) en Canarias vienen a poner de manifiesto, la viabilidad de este cultivo en invernadero y la posibilidad de su mayor expansión a corto plazo, debido a la introducción a lo largo de los últimos años de cultivares comerciales y de gran calidad, procedentes de Hawaii ('Sunrise') y de Brasil 'Baixinho de Santa Amalia' (BSA). En este trabajo se ha estudiado el crecimiento, desarrollo y productividad de los cultivares Sunset, Sunrise, y de los genotipos BH-65 y 'Baixinho de Santa Amalia' y las características morfológicas y organolépticas de sus frutos, con el fin de continuar con la selección de aquellos cultivares que mejor se adapten y cumplan con las exigencias mínimas de mercado, que mantengan una buena productividad en aquellas zonas marginales para la platanera y que sirvan de base para futuros programas de mejora. Los resultados ponen de manifiesto que los mayores porcentajes de floración se obtienen en el mes de Mayo (6 meses después de la plantación) en las plantas hermafroditas de todos los cultivares, correspondiendo los mayores porcentajes a 'Sunset' y 'Sunrise' ( 80 y 90,4\%, respectivamente). El número de hojas emitidas mensualmente es semejante en todos los cultivares, entre 7,5 y 7,8. Las plantas femeninas de 'Sunrise' así como las hermafroditas de 'Sunset' tienen mayor diámetro, y además, este último cultivar es más precoz en florecer y sus frutos son de mayor peso y tamaño. Hay que destacar también que en el punto de consumo los frutos del cultivar Sunrise son los que poseen mayor contenido en sólidos solubles totales (10,33 $\left.{ }^{\circ} \mathrm{Brix}\right)$. Además, los frutos hermafroditas de 'Sunrise' son los primeros en ser recolectados (422 días desde la plantación). Finalmente, señalar que el cultivar Sunset destaca por ser el más productivo $(59,5 \mathrm{~kg} / \mathrm{pl})$ y por tener mayores rendimientos por hectárea $(158,627 \mathrm{~kg} / \mathrm{ha})$. No obstante, los genotipos BH-65 y BSA poseen un comportamiento muy semejante, si bien tienen menor porte en el primer año de cultivo (142,9 y $138,8 \mathrm{~cm}$ ) menor altura a la primera flor $(40,6$ y $42,2 \mathrm{~cm}$ ) y menor longitud de entrenudos $(1,4$ y $1,3 \mathrm{~cm})$.Las características descriptivas de los frutos son muy parecidas sin existir diferencias significativas entre ellas. Los frutos tienen buen tamaño $(13,74$ x 7,81 cm y $(12,68$ x 7,98 cm) y peso $(392,92$ y 418,8 g.) y una producción excelente, cualidades de gran interés para el cultivo de la papaya bajo invernadero.

Palabras clave: Carica, fenología, características organolépticas, producción, rendimiento.

\section{BEHAVIOR OF PAPAYA CULTIVARS SUNSET, SUNRISE AND GENOTYPES OF 'BAIXINHO DE SANTA AMALIA'AND BH - 65 IN THE SOUTH OF TENERIFE ISLAND}

\begin{abstract}
The history of the papaya (Carica papaya L.) in the Canary Islands came to show the viability of this crop under greenhouse and the possibility of further expansion in short term due to the introduction, over the last years, of high-quality of commercial cultivars from Hawaii ('Sunrise') and Brazil 'Baixinho de Santa Amalia'(BSA). In this work it was studied the growth, development and productivity of the cultivars Sunset, Sunrise and the genotype BH65, and 'Baixinho de Santa Amalia', and the morphological and organoleptic characteristics of the fruits, in order to continue with the selection of those cultivars that best adapted and agree with the market requirements, maintain a good productivity in marginal areas for banana production and serve as a basis for future breeding programs. The results show that the highest flowering percentages took place in May (6 months after being planted) in hermaphrodite plants for all cultivars, corresponding to 'Sunset' and 'Sunrise' the maximum percentages ( 80 and $90.4 \%$, respectively). The number of leaves emitted monthly is similar in all cultivars, between 7.5 and 7.8. The female plants of 'Sunrise'and the hermaphrodites of 'Sunset' have greater diameter, and in addition, the last cultivar is precocious in blooming and the fruits are bigger in size and weight. Note also that at the consumption point of the fruit, the cultivar Sunrise showed the highest total soluble solids content $\left(10.33^{\circ}\right.$ Brix $)$. Moreover, hermaphrodite fruits of 'Sunrise' are the first to be collected (422 days from planting). Finally, the cultivar Sunset has become the most productive $(59.5 \mathrm{~kg} / \mathrm{pl})$ and has the highest yields per hectare $(158.627 \mathrm{~kg} / \mathrm{ha})$. However, the genotypes BH-65 and BSA have a very similar behaviour, but are smaller in the first year of cultivation $(142.9$ y $138.8 \mathrm{~cm})$ the first flower appears at a lower height (40.6 y 42.2 $\mathrm{cm})$ and a there is lower length of internodes $(1.4 \mathrm{y} 1.3 \mathrm{~cm})$. The descriptive characteristics of the fruits are very similar with no significant differences between them. The fruits have a good size $(13.74 \times 7.81$ y $12.68 \times 7.98 \mathrm{~cm})$ and weight (392.92 and $418.8 \mathrm{~g}$.) and excellent production qualities of great interest for the cultivation of papaya under greenhouse. Index terms: Carica, phenology, organoleptic characteristics, production performance.
\end{abstract}

(Trabalho 102-09). Recebido em: 24-04-2009. Aceito para publicação: 14-10-2010.

${ }^{2}$ Doctora en Biología. Investigadora del Departamento de Fruticultura Tropical. Instituto Canario de Investigaciones Agrarias. Apdo. 60. 38200 La Laguna. Tenerife. Islas Canarias. E-mail:crpastor@icia.es

${ }^{3}$ Doctora en Farmacia. Investigadora del Departamento de Fruticultura Tropical.Instituto Canario de Investigaciones Agrarias. Apdo. 60. 38200 La Laguna. Tenerife. Islas Canarias. E-mail: globo@icia.es

${ }^{4}$ Doctora en Biología. Departamento de Suelos y Riegos. .Instituto Canario de Investigaciones Agrarias. Apdo. 60. 38200 La Laguna. Tenerife. Islas Canarias. E-mail:clsuárez@icia.es 


\section{INTRODUCCIÓN}

En los últimos años como ya se ha mencionado anteriormente, se han introducido y evaluado en Canarias por el Departamento de Fruticultura Tropical, cultivares comerciales de papaya procedentes de Hawai ('Sunrise' 'Waimanalo', 'Higins', 'Kapoho') (RODRÍGUEZ PASTOR, 1989) y de Brasil ('Baixinho de Santa Amalia') Rodríguez Pastor, (2002), así como la puesta a punto de distintas técnicas de cultivo, lo cual ha influido enormemente en la expansión que ha experimentado dicho cultivo en las islas. Esta expansión viene asimismo acompañada a su vez, por el aumento del consumo de papaya registrado en los últimos años, debido principalmente, a las características nutritivas y medicinales de esta fruta, coincidiendo con lo mencionado por Tecson-Mendoza, (2007) y Corral-Aguayo et al., (2008). Estos cultivares han resultado ser muy interesantes para sustituir cultivos de invernadero tanto de platanera como de hortalizas, (GALÁN SAÚCO y RODRÍGUEZ PASTOR, 2007), adaptándose satisfactoriamente a las condiciones climáticas subtropicales de las islas.

La introducción de nuevos cultivares comerciales ha tenido como objetivo principal y mediante la aplicación de nuevas tecnologías la selección de cultivares con menor porte, baja carpeloidía, mayor porcentaje de sólidos solubles, alta productividad así como frutos de tamaño pequeño que son los requeridos en el mercado de exportación. Hasta el momento el cultivar Baixinho de Santa Amalia (BSA) ha resultado ser más interesante, Rodríguez Pastor, (2002). Es una mutación natural de 'Sunrise', originada en Brasil, de porte bajo y entrenudos cortos. La altura de inserción de las flores está entre 50 y $70 \mathrm{~cm}$. s.n.s. Inicia la producción a los 8-9 meses de plantada. Produciendo $50-60 \mathrm{tm} / \mathrm{ha}$. Sus frutos pesan $550 \mathrm{~g}$, con la pulpa de color rojo - anaranjada (MARÍN et al., 1995).

Sin embargo, el gran número de flores emitidas por nudo representa un inconveniente importante, ya que se va a producir posteriormente apiñamiento de los frutos con el consiguiente deterioro de éstos y a su vez, aumento del coste de la mano de obra para realizar la práctica de aclareo. A su vez el cultivar Sunrise, es el resultado de un cruce de la línea 9 (Hawaii) y Kariya (Oahu). Es un cultivar precoz, de porte alto y productivo. Los frutos tienen un peso medio de $450 \mathrm{~g}$. Color de la pulpa rojo - naranja, piel lisa y firme. Los frutos hermafroditas son piriformes. La altura de inserción de las primeras flores está entre 70 y $80 \mathrm{~cm}$ s.n.s. y comienza la recolección a los 9 - 10 meses de la plantación, con una producción en torno a los $45 \mathrm{tm} / \mathrm{ha}$ en el primer año y $39 \mathrm{tm} / \mathrm{ha}$ en el segundo año, (MARÍN et al., 1995). Su porte alto, va a determinar que la recolección de la fruta se haga con mayor dificultad y coste de mano de obra. Igualmente le ocurre al cultivar Sunset, el cual tiene un porte alto, y productivo. Los frutos tienen un peso medio de $400 \mathrm{~g}$. La pulpa es de color rojo - anaranjada de piel lisa, firme, resistente al transporte y de larga vida comercial. La inserción de las primeras flores está entre 60 y $70 \mathrm{~cm}$ s.n.s. Produce de media $40 \mathrm{tm} /$ ha con el inicio de la recolección a los 8 meses de realizada la plantación. (MARÍN et al., 1995). A su vez, el genotipo BH-65 es una selección realizada en Sudáfrica a partir del cultivar Baixinho de Santa Amalia, que ha dado lugar a este nuevo híbrido (Aart Louw, comunicación personal). De porte bajo, con entrenudos más cortos pero menos flores por nudo que el cultivar Baixinho de Santa Amalia. La inserción de las flores es a $40-60 \mathrm{~cm}$ s.n.s. El fruto pesa 452 g. y tiene menos manchas de origen fisiológico (skin freckles) que otros cultivares. La cosecha potencial así como las características del fruto son semejantes a 'Sunrise' Solo. Tiene mejor cuajado que 'Sunrise' a temperaturas bajas. El objetivo de este trabajo es la evaluación del cultivar de papaya 'Sunset' y del genotipo BH-65 y conocer su comportamiento comparativamente con los ya evaluados anteriormente, lo que nos permitirá iniciar programas de mejora genética en un futuro próximo.

\section{MATERIAL Y MÉTODOS}

El ensayo se ha realizado en invernadero de malla de $2.400 \mathrm{~m}^{2}$ perteneciente al Instituto Canario de Investigaciones Agrarias (I.C.I.A.) ubicado en la Finca La Planta, a 115 m s.n.m. en la localidad de Güimar, a una longitud de $16^{\circ} 23^{\prime}$ y a una latitud de $28^{\circ} 19^{\prime}$ en la vertiente sur de la isla de Tenerife La duración del estudio abarcó 2 años y medio, desde Noviembre de 2005 a Mayo de 2008.

El diseño experimental ha sido bloques al azar. El número de tratamientos fué 4, cuatro cultivares de papaya: 'Sunset' (Improved Sunrise Solo ó línea 72/12), 'Sunrise', ‘BH-65’ y ‘Baixinho de Santa Amalia'.

Cada unidad experimental constó de 24 plantas, las cuales las laterales, fueron consideradas plantas bordes y las 8 centrales se usaron para la toma de datos. El número de repeticiones fue de 4 . El marco de plantación, 2,5 x 1,5 m (2.666 pl/ha). Para el tratamiento estadístico de los datos se usó el programa SPSS y SYSTAT 10, realizándose un ANOVA y análisis de la varianza y el test de comparación de medias Tukey para $\mathrm{p} \leq 0,05$ y el test de 
significancia de Pearson.

\section{Parámetros a tomar en la planta:}

Altura total del tallo ( $1^{\text {er }}$ año), medida desde el suelo hasta el ápice de la planta. Altura de la primera flor, medida desde el suelo hasta la aparición de la primera flor. Diámetro del tallo a la altura de la primera flor. Fecha de floración. Intervalo de la floración - recolección. Se estima, contando el $n^{\circ}$ de días que transcurren desde la floración de cada planta hasta el inicio de recolección de cada una de ellas. Ritmo de emisión de hojas/mensual. Se contaron las hojas nuevas emitidas mensualmente, desde la última marcada con $2 \mathrm{~cm}$ de longitud. $\mathrm{N}^{\circ}$ de nudos, comprendidos en una distancia predeterminada en el tallo (1m). Longitud del entrenudo, se determina en base al cociente entre una distancia elegida a lo largo del tallo de la planta $1 \mathrm{~m}$ en este caso y el número de nudos existentes en esa distancia. Fecha del inicio de la recolección. Número de frutos/planta/año. Número de Kg/planta/año. Porcentaje de carpeloidía.

\section{Parámetros a tomar en el fruto.}

Longitud y Anchura. Ambos parámetros se miden en cm cortando la fruta por la mitad en sentido longitudinal. La longitud, midiendo el fruto desde la base hasta el ápice y la anchura, midiendo la zona ecuatorial del fruto de un extremo a otro. Firmeza, expresada en Newtons se midió con un penetrómetro de la marca Chatillon ${ }^{\circledR}$ modelo DDP $5 \mathrm{~kg}$. Grosor de la pulpa en $\mathrm{mm}$, se realizan las medidas con un calibrador o pie de rey. Color de la pulpa y de la piel según escala de color de Lam, citado por Chan et al., 1994. Porcentaje de manchas en la piel, se realiza la medición mediante una escala prefijada que va de de 0 a 5 (Eloísa et al., 1994) y está referida al porcentaje de área que ocupan las manchas en la superficie de la piel del fruto. Sólidos solubles totales, ( ${ }^{\circ}$ Brix) realizada con un refractómetro de compensación automática Atago $^{\circledR}$, modelo ATC-1. Peso del fruto (g), se realizó individualmente en cada muestreo realizado.

\section{RESULTADOS Y DISCUSIÓN}

\section{Parámetros morfológicos y fenológicos}

Los cultivares Sunset y Sunrise presentan mayor longitud del entrenudo que el resto (Figura 1), $(3,6$ y $3,2 \mathrm{~cm})$ lo que no es una ventaja, ya que si bien impide que los frutos se rocen unos con otros, son por otra parte más altos $(339,2$ y $284 \mathrm{~cm})$ respectivamente, estando la altura más relacionada con la longitud del entrenudo que con el número de nudos (NAKASONE and STOREY, 1955), aunque esto no repercute en el número de hojas emitidas. Si bien, en condiciones tropicales, los cultivares que más hojas emitieron fueron 'Sunset' y BH-65 (ALONSO et al.,
2008 a). Hay que recordar que el ensayo está realizado en invernadero donde la merma de luz es evidente, por lo que la altura que alcanzan las plantas será mayor por la estimulación del heliotropismo (PERES et al., (2008) (Figura 1). Este factor no es deseable ya que las plantas se ahilan demasiado y la recolección de la fruta tiene mayor dificultad y costes.

No existen diferencias significativas entre los diámetros de las plantas femeninas y hermafroditas a la altura de la primera flor de los genotipos BH-65 y BSA y los cvs. Sunset y Sunrise. Sin embargo, se destaca el cultivar Sunset por tener el mayor diámetro de tallo en las plantas hermafroditas al inicio de la recolección respecto al resto de los cultivares, lo que podría estar relacionado con la máxima producción de este cultivar como se verá más adelante.

La altura de las plantas del cultivar Sunset al año de la plantación, difiere significativamente del resto de cultivares estudiados, no observándose diferencias entre las plantas hermafroditas y femeninas (Figura 2).

El número de hojas emitidas mensualmente (entre 7,5 y 7,8 hojas en media), es semejante para todos los cultivares, produciéndose un aumento en el número de hojas emitidas semanalmente en todos ellos (Figura 3), en los meses de junio, julio y agosto, $\left(22.5^{\circ} \mathrm{C}-24^{\circ} \mathrm{C}-24^{\circ} \mathrm{C}\right)$ cuando las temperaturas comienzan su ascenso coincidiendo ello con lo expuesto por Peres et al., (2008), al cultivar la papaya en invernadero. Hay que señalar al respecto, que los resultados obtenidos en los meses más calurosos en Canarias, se aproximan a los obtenidos en Hawai en los meses fríos (2,4 hojas/semana) (NAKASONE; PAULL 1998)

La altura en que se emite la primera flor en los cultivares estudiados tiene un comportamiento decreciente: 'Sunset' > 'Sunrise' > BSA y BH-65 (RANCEL, 2006). Siempre es menor tanto para las plantas hermafroditas como para las femeninas en los genotipos BH-65 y BSA (Figura 4). Es posible que esta diferencia tan señalada sea debido a las circunstancias climatológicas especiales que ocurrieron en el mes de noviembre de 2005 en Canarias (ciclón Delta) que arrasó el techo del invernadero, dejó al descubierto las plantas recién plantadas y éstas sufrieron una considerable ralentización en el crecimiento y desarrollo hasta el mes de marzo de $2006\left(19^{\circ} \mathrm{C}\right)$. Se trató de relacionar también la altura de la primera flor con la altura de la planta y sólo se obtuvo una buena relación para el genotipo BSA $\left(\mathrm{R}^{2}=0,631, \mathrm{P}=0,000, \quad \mathrm{~N}=23\right)$ coincidiendo con los resultados descritos por Alonso et al., (2008 a).

Debido a las condiciones climatológicas 
adversas que ya se han mencionado anteriormente, los mayores porcentajes de floración se obtienen en el mes de Mayo de 2006 ( $\left.22^{\circ} \mathrm{C}\right)$ en las plantas hermafroditas de todos los cultivares estudiados, (6 meses después de la plantación), hecho poco habitual, ya que lo normal es dos meses después. Obteniendo los mejores resultados, los cultivares Sunset y Sunrise ( $80 \%$ y $90,4 \%$ respectivamente).

\section{Ciclo de cultivo}

Se observa que el ciclo de cultivo se ha alargado ligeramente. El intervalo desde la plantación hasta el inicio de la recolección en las condiciones del ensayo ha sido de 14 meses, cuando en condiciones normales está entre 9 y 12 meses (RODRÍGUEZ PASTOR, 2002). También hay retraso en la floración (4 meses) como ya se ha puesto de manifiesto anteriormente.

Los resultados obtenidos ponen de manifiesto que no existen diferencias significativas entre los cultivares estudiados (Figura 5). Estos valores son superiores a los observados cuando se inicia la floración en verano, (RANCEL, 2006). Hay que destacar que en este caso las plantas hermafroditas de todos los cultivares florecieron antes que las femeninas, contrariamente a lo que ha ocurrido en otros ensayos.

Según los valores mostrados en la Figure 6 en los que tampoco se aprecian diferencias significativas entre cultivares, se puede decir que el hecho de que los cultivares sean más precoces en florecer no significa que sus frutos sean los primeros en recolectarse.

Asimismo, esta variabilidad de los cultivares en el intervalo floración - recolección, va a depender de varios factores como: cultivar, época del año de la plantación, grado de color al recolectarse el fruto (NAKASONE; STOREY, 1955).

\section{Características descriptivas de los frutos} hermafroditas y femeninos

Según la Tabla 1, el cultivar que mayor peso obtuvo en sus frutos procedentes de plantas hermafroditas fue 'Sunset' $(498,13$ g.) y con mayores dimensiones y firmeza a excepción de 'Sunrise'. De todas formas, el peso de los frutos procedentes de las plantas hermafroditas del resto de los cultivares está dentro de los límites establecidos para la comercialización. Sin embargo el mayor peso de los frutos de las plantas femeninas correspondió a los genotipos BSA y BH-65 (244,85g. y 231,61g.) respectivamente, sin que hubiese diferencias sig- nificativas entre ellos, teniendo ambos mayor firmeza que el resto. Destacando, que el tamaño menor de los frutos femeninos se debe principalmente, a que en invernadero, la polinización es escasa o nula debido a la falta de ventilación, que favorece el transporte del polen y no llega a producirse la fecundación, por lo que los frutos ven disminuido su tamaño (RODRÍGUEZ PASTOR et al., 1990) y son generalmente partenocárpicos. Sin embargo, esto no ocurre en los frutos procedentes de flores hermafroditas, ya que éstas son cleistógamas, (DAMASCENO JUNIOR et al., 2009) y por ello, la polinización está asegurada.

En cuanto al grosor de la pulpa, no existen diferencias significativas entre cultivares, respecto a los frutos hermafroditas. No obstante, en los frutos de flores femeninas, sí se detectan diferencias en el grosor, siendo mayores para los genotipos BH-65 y BSA y menor para el cultivar Sunrise. El cultivar Sunset destaca por tener los frutos hermafroditas con mayor anchura que el resto de cultivares. En lo referente a la longitud de éstos, no se observó diferencia significativa alguna.

Respecto al color de la pulpa, de la piel y porcentaje de manchas, no existen diferencias entre ellos, pues son todos los cultivares de color naranja y con el mismo porcentaje de manchas (25\%). Estas manchas, se manifiestan en mayor grado en la cara de los frutos expuesta a la luz solar directa, (REIS et al., 2008) siendo su causa atribuida a factores abióticos (LIBERATO; ZAMBOLIN, 2002). Otros estudios concluyen, que las condiciones ambientales y culturales (MARELLI et al., 2008; GOMES et al., 2008), influyen y además, se observa en cortes histológicos de los frutos, las paredes engrosadas de los tejidos, por la influencia del látex derramado por los vasos laticíferos (FRAGA, 2001). Estas condiciones ambientales así como las prácticas culturales pueden incidir también, en otras especies como el níspero (Eribotrya japonica, L.), ocasionando la aparición de la mancha púrpura, (GARIGLIO et al., 2008 a y b).

Los frutos hermafroditas del genotipo BSA destacan por tener menor contenido en sólidos solubles totales $\left(9,13^{\circ}\right.$ Brix) existiendo diferencias significativas respecto a los otros cultivares. Hay que señalar, que no se obtuvieron diferencias significativas entre cultivares, respecto a los frutos de flores femeninas. Estos resultados podían haber sido superiores, pero es posible sea debido en primer lugar a que las plantas estuvieron durante un período bastante largo (5 meses) con las hojas secas, no funcionales, por causas imprevistas y en segundo lugar porque la recolección se inició en enero de $2007\left(15^{\circ} \mathrm{C}\right)$ y en los meses anteriores, últimos meses de desarrollo del fruto, es cuando el azúcar aumenta rápidamente (NA- 
KASONE; PAULL 1998) y si las temperaturas son bajas, $\left(17^{\circ} \mathrm{C}\right)$ el contenido de sólidos solubles totales disminuirá también. En otros ensayos realizados en la misma zona y en épocas diferentes (RODRÍGUEZ PASTOR, 2002), el genotipo BSA ha dado mejores resultados $\left(11^{\circ}\right.$ Brix $)$.

Respecto a la firmeza de los frutos, se observa en la tabla 1 que siendo uno de los parámetros de calidad más importantes en la papaya, los valores más altos correspondieron a los frutos femeninos de los genotipos BH-65, BSA y 'Sunrise'. Para los frutos hermafroditas, el mayor valor fue para el cultivar Sunrise. Rancel et al., (2007), muestran que la firmeza en los frutos hermafroditas del genotipo BSA es alta (8.39) respecto al genotipo BH-65 (3.27) y al cultivar Sunset (3.4), aunque la pérdida de firmeza desciende de forma gradual en el genotipo BSA (RANCEL, 2006). Sin embargo, en 'Sunset' y BH65 desciende en principio bruscamente (pérdida del $95 \%$ de su firmeza inicial), manteniéndose constante en el punto de consumo.

En cuanto al porcentaje de carpeloidía, todos los cultivares tienen menos del 5\% lo que indica una buena adaptación a las condiciones del medio, a excepción del cultivar Sunset que posee un porcentaje muy superior $(16 \%)$ lo que se podría explicar si se tiene en cuenta que el diámetro es un indicador del vigor de las plantas y 'Sunset' tiene el mayor diámetro de tallo por lo tanto, mayor vigor, y éste está relacionado también con la carpeloidía (tabla 2).

\section{Producción y Rendimientos}

Los cultivares Sunset y Sunrise tuvieron los mayores valores respecto al número de frutos por planta de flores hermafroditas (141,5 y 138,5 frutos/planta) respectivamente, y para frutos de flores femeninas, los mayores valores correspondieron a BSA y 'Sunrise' (214/planta y 231/planta) respectivamente. Siendo el cultivar Sunset el que obtuvo mayor número de kilos de fruta, de flores hermafroditas por planta $(59,5 \mathrm{~kg})$, además de tener el menor número de frutos $<$ de $200 \mathrm{~g}$ (tabla 2) lo que constituye una ventaja frente al resto de cultivares, ya que ese tamaño no es considerado comercial en el mercado de exportación (NAKASONE; PAULL, 1998). Sin embargo hay que destacar que es el genotipo BSA el que más kilos de fruta procedente de flores femeninas produce ( $42 \mathrm{~kg} /$ planta) existiendo diferencias significativas sólo con el cultivar Sunrise (Tabla 2).

También, se obtuvo una buena correlación entre la producción por planta de los cultivares, Sunset, Sunrise y el genotipo BSA y el diámetro del tallo de dichas plantas $\left(\mathrm{R}^{2}=0,744 ; \mathrm{R}^{2}=0,713\right.$ y $\mathrm{R}^{2}=$ $0,731)$ respectivamente. Hay que destacar que 'Sunset', produjo mayores rendimientos por hectárea en cuanto a las plantas hermafroditas y el genotipo BSA respecto a las femeninas (Tabla 3 )

TABLA 1 - Características descriptivas de los frutos hermafroditas y femeninos de los cultivares de papaya de la isla de Tenerife, 2005-2008.

\begin{tabular}{|c|c|c|c|c|c|c|c|c|}
\hline \multirow{3}{*}{$\begin{array}{c}\text { Características } \\
\text { descriptivas }\end{array}$} & \multicolumn{8}{|c|}{ Cultivar y Sexo } \\
\hline & \multicolumn{2}{|c|}{ BSA } & \multicolumn{2}{|c|}{ BH-65 } & \multicolumn{2}{|c|}{ SUNSET } & \multicolumn{2}{|c|}{ SUNRISE } \\
\hline & herm. & fem. & herm. & fem. & herm. & fem. & herm. & fem. \\
\hline Peso (g) & $418,80 \mathbf{b}^{1}$ & $244,85 \mathbf{a}$ & $392,92 \mathbf{b}$ & $231,61 \mathbf{a}$ & $498,13 \mathbf{a}$ & $207,00 \mathbf{b}$ & $398,35 \mathbf{b}$ & $183,17 \mathbf{c}$ \\
\hline Longitud (cm) & $12,68 \mathbf{a}$ & $10,58 \mathbf{a}$ & $13,64 \mathbf{a}$ & $11,02 \mathbf{a}$ & $14,00 \mathbf{a}$ & $9,15 \mathbf{a b}$ & $12,26 \mathbf{a}$ & $8,66 \mathbf{b}$ \\
\hline Anchura (cm) & $7,98 \mathbf{b}$ & $7,33 \mathbf{a}$ & $7,81 \mathbf{b}$ & $7,17 \mathbf{a}$ & $8,38 \mathbf{a}$ & $6,96 \mathbf{b}$ & $7,87 \mathbf{b}$ & $6,71 \mathbf{b}$ \\
\hline Grosor (cm) & & & & $1,43 \mathbf{a}$ & $1,88 \mathbf{a}$ & $1,36 \mathbf{b}$ & $1,83 \mathbf{a}$ & $1,29 \mathbf{c}$ \\
\hline Color Pulpa & naranja & naranja & naranja & naranja & naranja & naranja & naranja & naranja \\
\hline Color Piel & naranja & naranja & naranja & naranja & naranja & naranja & naranja & naranja \\
\hline Manchas (\%) & 25 & 25 & 25 & 25 & 25 & 25 & 25 & 25 \\
\hline TSS ( ${ }^{\circ}$ Brix) & $9,13 \mathbf{b}$ & $10,23 \mathbf{a}$ & $9,60 \mathbf{a}$ & $10,66 \mathbf{a}$ & $9,65 \mathbf{a}$ & $10,17 \mathbf{a}$ & $10,33 \mathbf{a}$ & $10,34 \mathbf{a}$ \\
\hline Firmeza $\left(\mathrm{kg} / \mathrm{cm}^{2}\right)$ & $4,96 \mathbf{b}$ & $7,86 \mathbf{a}$ & $5,38 \mathbf{b}$ & $7,57 \mathbf{a}$ & $5,55 \mathbf{a b}$ & $5,71 \mathbf{b}$ & $7,01 \mathbf{a}$ & $7,28 \mathbf{a}$ \\
\hline
\end{tabular}

${ }^{1}$ Las cifras de cada tratamiento difieren cuando no van seguidas de la misma letra, según el test de diferencias significativas de Tukey $(\mathrm{p} \leq 0,05)$. 
TABLA 2 - Características productivas de las plantas hermafroditas y femeninas de los cultivares de papaya de la isla de Tenerife, 2005-2008.

\begin{tabular}{|c|c|c|c|c|c|c|c|c|}
\hline \multirow{3}{*}{$\begin{array}{c}\text { Características } \\
\text { productivas }\end{array}$} & \multicolumn{8}{|c|}{ Cultivar y Sexo } \\
\hline & \multicolumn{2}{|c|}{ BSA } & \multicolumn{2}{|c|}{ BH-65 } & \multicolumn{2}{|c|}{ SUNSET } & \multicolumn{2}{|c|}{ SUNRISE } \\
\hline & herm. & fem. & herm. & fem. & herm. & fem. & herm. & fem. \\
\hline $\mathbf{N}^{0}$ Frutos/pl. & $114,0 \mathbf{b}^{1}$ & $214,0 \mathbf{a}$ & $126,5 \mathbf{a b}$ & $188,5 \mathbf{a b}$ & $141,5 \mathbf{a}$ & $138,0 \mathbf{b}$ & $138,5 \mathbf{a}$ & $231 \mathbf{a}$ \\
\hline Peso (kg/pl.) & $35,5 \mathrm{c}$ & $42,0 \mathbf{a}$ & 38,5 bc & $37,0 \mathbf{a b}$ & $59,5 \mathbf{a}$ & $25,0 \mathbf{b}$ & $44,5 \mathbf{b}$ & $35,5 \mathbf{a b}$ \\
\hline pl carpeloides (\%) & $2 \mathbf{b}$ & n.e & $0,5 \mathbf{b}$ & n.e & $16,0 \mathbf{a}$ & n.e & $2,0 \mathbf{b}$ & n.e \\
\hline $\mathbf{N}^{0}$ Frutos deformes/pl. & $11,5 \mathbf{a}$ & $12,0 \mathbf{a}$ & $10,5 \mathbf{a b}$ & $19,0 \mathbf{a}$ & 6,0 bc & $6,0 \mathbf{a}$ & $4,5 \mathbf{c}$ & $16,0 \mathbf{a}$ \\
\hline $\mathrm{N}^{0}$ Frutos $(<200 \mathrm{~g} / \mathrm{pl})$. & $14,0 \mathbf{a}$ & $100,5 \mathbf{b}$ & $19,5 \mathbf{a}$ & 84,0 b & $13,5 \mathbf{a}$ & $76,0 \mathbf{b}$ & $26,0 \mathbf{a}$ & $154,5 \mathbf{a}$ \\
\hline
\end{tabular}

${ }^{1}$ Las cifras de cada tratamiento difieren cuando no van seguidas de la misma letra, según el test de diferencias significativas de Tukey $(\mathrm{p} \leq 0,05)$. n.e. (no existe).

TABLA 3 - Producción total y Rendimientos por hectárea de los distintos cultivares de papaya.

\begin{tabular}{lcccccccc}
\hline Cultivar & \multicolumn{2}{c}{ BSA } & \multicolumn{2}{c}{ BH-65 } & \multicolumn{2}{c}{ SUNSET } & \multicolumn{2}{c}{ SUNRISE } \\
\hline Sexo & herm. & fem. & herm. & fem. & herm. & fem. & herm. & fem. \\
Producción total (kg) & 1.190 & 368 & 1.234 & 306 & 1.556 & 229 & 1.380 & 381 \\
N $^{\mathbf{0}}$ Frutos totales & 2.848 & 1.500 & 3.141 & 1.319 & 3.125 & 1.104 & 3.469 & 2.081 \\
Rendimiento ha. (kg) & 94.643 & 111.972 & 102.641 & 98.642 & 158.627 & 66.650 & 118.637 & 93.310 \\
\hline
\end{tabular}

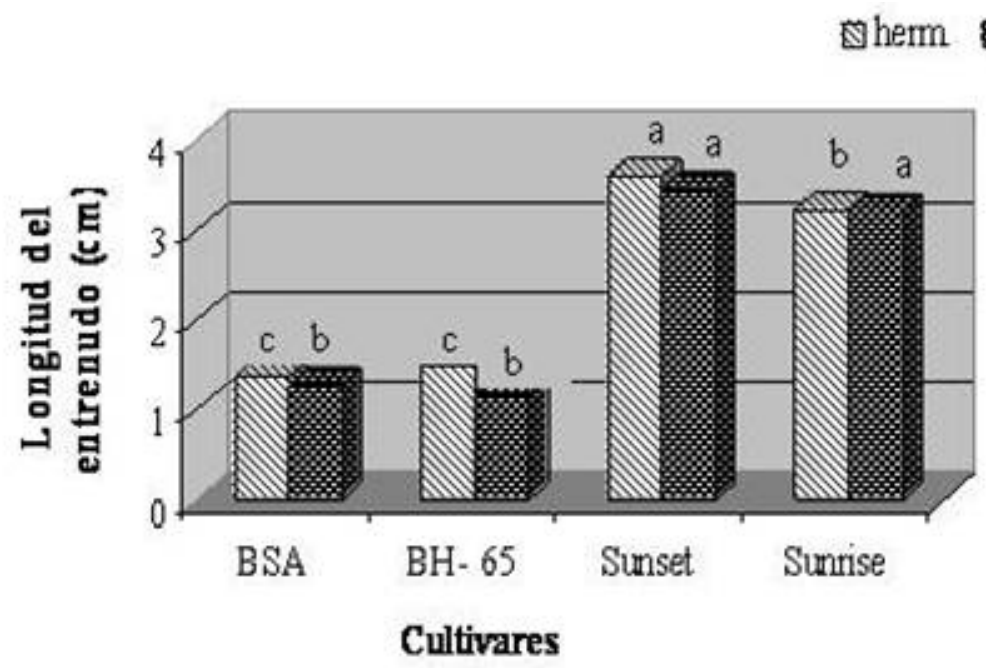

FIGURA 1 - Longitud del entrenudo de las plantas hermafroditas y femeninas de los diferentes cultivares.

Las cifras de cada tratamiento difieren significativamente cuando no van seguidas por la misma letra, según el test de diferencias significativas de Tukey $(\mathrm{p} \leq 0,05)$. 


\section{$\mathbb{\$}$ herm 图 fem}

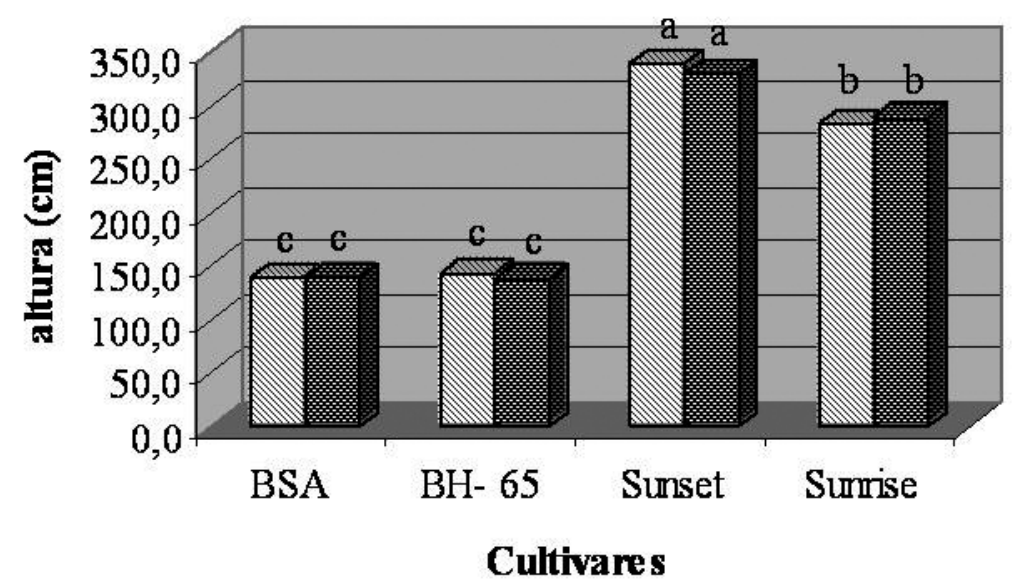

FIGURA 2 - Altura total de las plantas hermafroditas y femeninas al año de la plantación en los cultivares estudiados.

Las cifras de cada tratamiento difieren significativamente cuando no van seguidas por la misma letra, según el test de diferencias significativas de Tukey $(\mathrm{p} \leq 0,05)$.

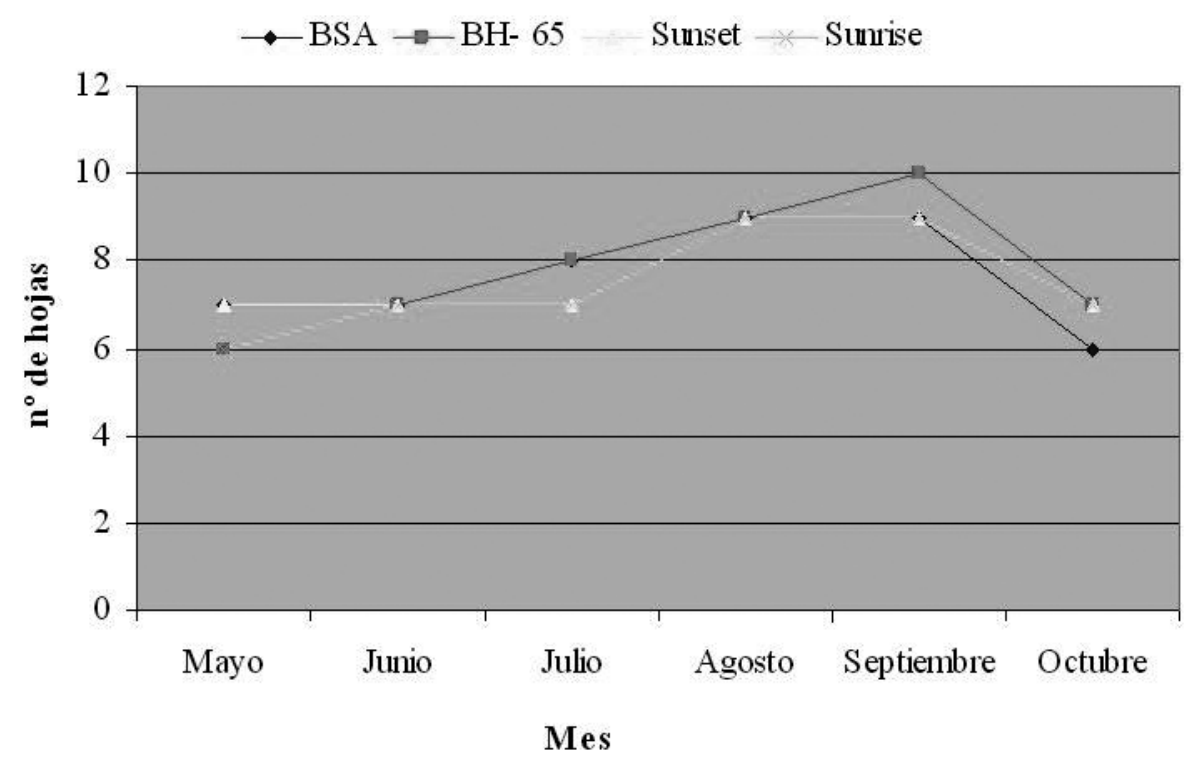

FIGURA 3 - Número de hojas emitidas por los diferentes cultivares en las condiciones experimentales.

Las plantas estuvieron durante un período largo (5 meses) con las hojas secas y no funcionales. 


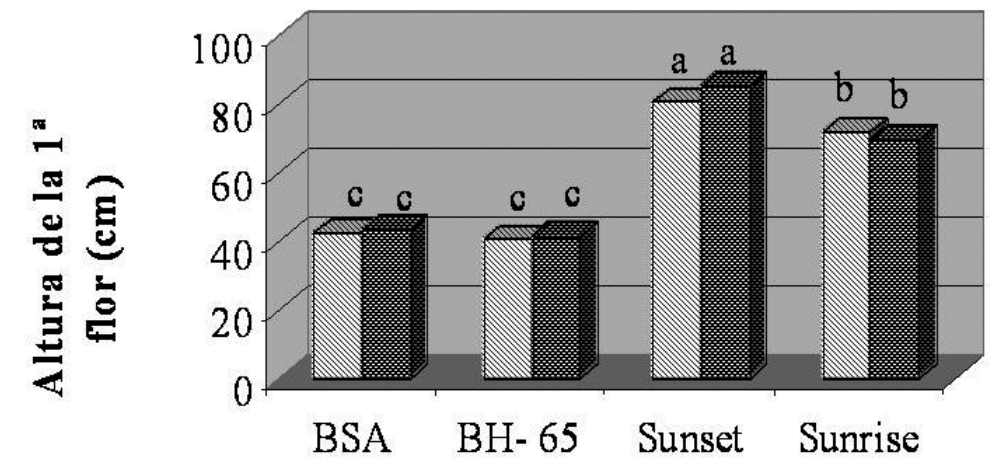

Cultivares

FIGURA 4 - Altura de la $1^{\text {a }}$ flor en las plantas hermafroditas y femeninas de los diferentes cultivares de papaya.

Las cifras de cada tratamiento difieren significativamente cuando no van seguidas de la misma letra, según el test de diferencias significativas de Tukey $(\mathrm{p} \leq 0,05)$.

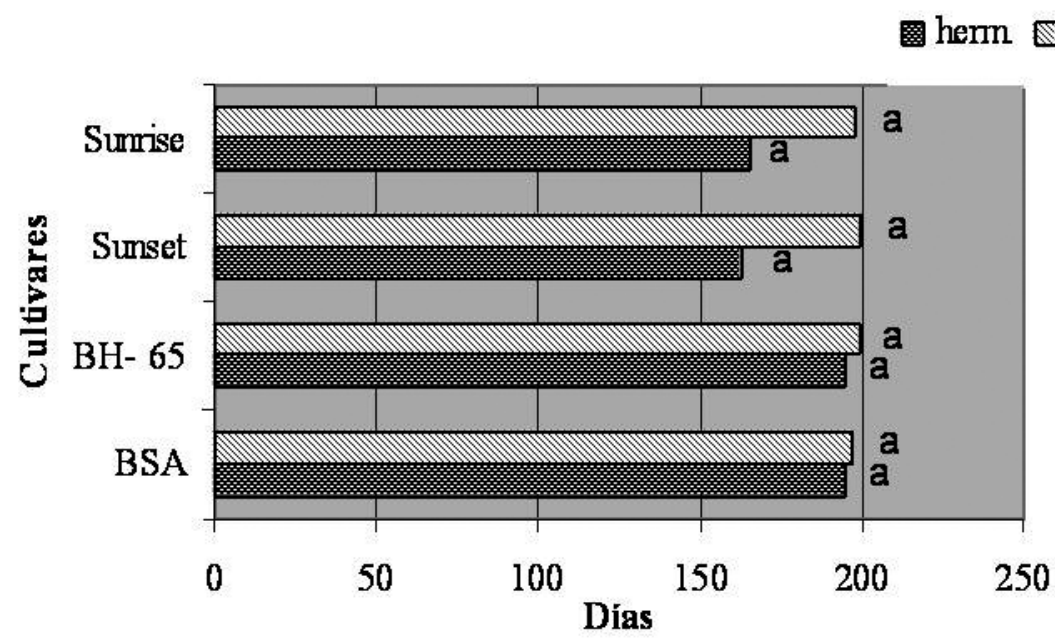

FIGURA 5 - Días transcurridos entre la plantación - floración en los diferentes cultivares de papaya.

Las cifras de cada tratamiento no difieren significativamente cuando van seguidas de la misma letra, según test de diferencias significativas de Tukey $(\mathrm{p} \leq 0,05)$. 


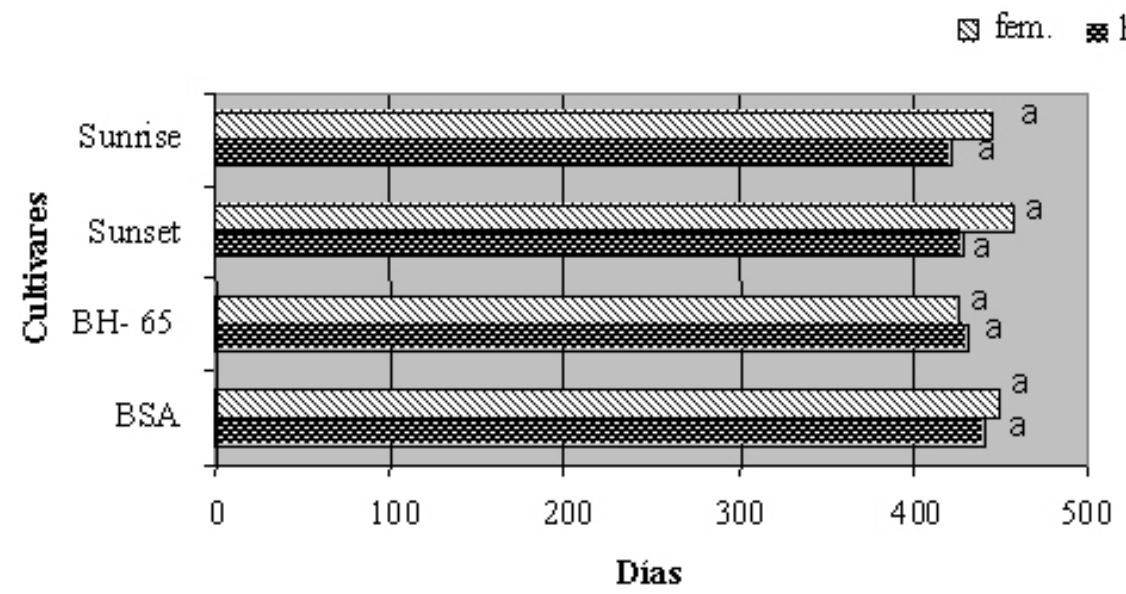

FIGURA 6 - Días transcurridos entre la plantación - recolección en los diferentes cultivares de papaya.

Las cifras de cada tratamiento no difieren significativamente cuando van seguidas de la misma letra, según test de diferencias significativas de Tukey $(\mathrm{p} \leq 0,05)$.

\section{CONCLUSIONES}

1 - Los cultivares Sunset y Sunrise tienen mayor longitud de entrenudos y mayor altura que el resto de cultivares, aunque existe el inconveniente de tener mayor dificultad en la recolección de los frutos.

2 - El diámetro del tallo de las plantas hermafroditas del cultivar Sunset es mayor que en el resto de cultivares, coincidiendo con la mayor producción.

3 - El número de hojas emitidas mensualmente en todos los cultivares es semejante, observándose un aumento de éstas cuando se elevan las temperaturas en verano.

4 - Existe una buena correlación entre la altura de la primera flor y la altura de las plantas sólo para el genotipo BSA.

5 - Los mayores porcentajes de floración se obtienen en el mes de Mayo de 2006 en todos los cultivares y las plantas hermafroditas florecen primero que las femeninas.

6 - Los frutos hermafroditas del cultivar Sunset tienen mayor peso y mayor tamaño que el resto. Sin embargo los frutos femeninos se destacan por su mayor tamaño, peso, firmeza y grosor que el híbrido BH-65 y el genotipo BSA.
7 - El cultivar BSA produce mayor número de $\mathrm{kg} /$ planta entre las plantas femeninas, y 'Sunset' entre las hermafroditas, existiendo además, una buena correlación entre la producción de las plantas y el diámetro del tallo de los cultivares Sunset, BSA y Sunrise al inicio de la recolección.

8 - Finalmente se puede concluir que, el cultivar Sunset es muy interesante en cuanto a producir mayor número de frutos de flores hermafroditas, de mayor peso y mayor número de kilos por planta. Es un cultivar interesante para explotaciones que deseen realizar plantaciones de papaya a un solo ciclo debido a la altura que alcanzan. Sin embargo, el cultivar BSA y el híbrido BH-65 poseen un comportamiento muy semejante, tienen menor porte en el primer año de cultivo, menor altura a la primera flor, y menor longitud de entrenudos, así como las características descriptivas de los frutos son muy parecidas sin que existan diferencias significativas entre ellas. Tienen también buen tamaño y peso del fruto y con una producción excelente, cualidades de gran interés para el cultivo de la papaya en invernadero. Además, todas estas cualidades repercuten finalmente en la economía del agricultor, ya que la recolección de la fruta se facilita al tener las plantas menor tamaño y existir menor coste de la mano de obra. 


\section{REFERENCIAS}

ALONSO, M.; TORNET, Y.; RAMOS, R.; FARRÉS, E.; CASTRO, J.; RODRÍGUEZ PASTOR, M. C. Evaluación de tres cultivares de papaya del grupo Solo basada en caracteres de crecimiento y productividad. Cultivos Tropicales, La Habana, v. 29, n. 2. p. 59-64, 2008 a.

CHAN, Y. K.; UTHAI, N.; WISNU, B.; KOAY, S. H.; ESPINO, R. C. Commercial papaya cultivars in Asean. In: ROHANI, M.D.Y. Papaya. Malasia: Ed. Food Technology Research Center, 1994. p. 5-17.

CORRAL-AGUAYO, R. D.; CARRILLO-LÓPEZ, A. Correlation between some nutritional components and the total antioxidant capacity measured with six different assays in eight horticultural crops. Journal of Agricultural and Food Chemistry, Washington, v.56, n. 22, p.10498-10504, 2008.

DAMASCENO JUNIOR, P.; SANTANA, T.; PEREIRA, M.; DA SILVA, F.; DE MAGALHAES, M.; RODRIGO, N. Preferencial reproduction mode of hermaphrodite papaya plant. Revista Brasileira de Fruticultura, Jaboticabal, v.31, n.1, p. 182-189, 2009.

ELOÍSA, M.; REYES, Q.; PAULL, R.3. Skin freeckles on solo papaya fruit. Scientia Horticulturae, Amsterdan, v. 58, p. 31-39, 1994.

FRAGA, F. J. Manchas en el fruto de la papaya (Carica papaya, L.). Caracterización y valoración de su incidencia. La Laguna, 2001. 108 f.. Trabajo (Fin de Carrera)- Centro Superior de Ciencias Agrarias, Universidad de La Laguna, Tenerife, 2001.

GALÁN, V.; RODRÍGUEZ PASTOR, M. C. Greenhouse cultivation of papaya. Acta Horticulturae, Lovaina, v.740, p. 191-197, 2007.

GARIGLIO, N. E.; REIG, C.; AGUSTÍ, M. Assimilate partitioning between the flesh and the rind is responsable for purple spot in loquat fruit. Journal of Horticultural Science, Kent, v. 83, n. 1, p. 3742, 2008 a.

GARIGLIO, N. E.; REIG, C.; MARTINEZFUENTES, A. Purple spot in loquat ( Eribotrya japonica, L.) is associated to changes in flesh-rind water during fruit development. Scientia Horticulturae, Amsterdan, v. 119, n. 1, p. 55-58, 2008 b.
GOMES, A.; DE OLIVEIRA, J.G.; VIANA, A. P.; PEREIRA, M.G. Skin freckles and yield components of papaya Tainung 01: effect of irrigation depths and soil coverings. Ciencia e Agrotecnologia, Lavras, v.32, n.4, p. 1161-1167, 2008.

LIBERATO, J.R.; ZAMBOLIM, L. Controle das doenças causadas por fungos, bactérias e nematóides em mamoeiro. In: ZAMBOLIM, L.; VALE, F.X.R.; MONTEIRO, A.J.A.; COSTA, H. (Ed.). Controle de doenças de plantas fruteiras. Viçosa: UFV, 2002. p.1023-1170

MARELLI, L. ; SILVA K.; PAES, B.; LOPES, SILVIO. L. Ascorbic acid, $\beta$-carotene and lycopene content in papaya fruits (Carica papaya) with or without physiological skin freckles. Scientia Agricola, Piracicaba, v.65, n.3, p. 246-250, 2008.

MARÍN, S. L.; GOMES, J.A.; SALGADO, J. S.; MARTINS, D.S.; FULLINE. A. Recomendações para a cultura do mamoeiro dos grupos Solo e Formosa no Estado do Espirito Santo. Vitória: EMCAPA, 1995. p. 16-17, 45-46, 57. (Circular Técnica, 3 )

NAKASONE, H.Y.; PAULL, R.E. Papaya. In: NAKASONE, H. Y.; PAULL, R. E. Tropical fruits. New York: CAb International, 1998. p. 239-257.

NAKASONE, H. Y.; STOREY, W.B. Studies on inheritance of fruiting height of Carica papaya, L. Proceeding American Society Horticultural Science, Hawaii, v.66, p.166-182, 1955.

PERES MARTELLETO, L.A.; RIBEIRO, R.; SUDO-MARTELLETO, M.; VASCONCELLOS, M.A.; MARIN, S.L.; PEREIRA, M., Cycle development and agronomic performance of organic papaya cultivation in protected environment. Revista Brasileira de Fruticultura, Jaboticabal, v. 30, n.3, p. 662-666, 2008.

RANCEL, J. Estudio sobre la fenología y poscosecha de la papaya (Carica papaya, L.) en los cultivares "Sunset", "Baixinho de Santa Amalia" y BH-65", bajo invernadero de malla en la vertiente sur de Tenerife. 2006. 377 f. Trabajo (Fin de Carrera) - Escuela Técnica Superior de Ingeniería Agraria, Universidad de La Laguna, Tenerife, 2006. RANCEL, J.; LOBO, G.; RODRÍGUEZ PASTOR, 
M. C. Postharvest behaviour of three cultivars under mesh greenhouse cultivation in Tenerife (Canary Islands). Acta Horticulturae, Wageningen, v.740, p. 295-302, 2007.

REIS, F.D., CAMPOSTRINI, E.; NETTO, A., Skin frecles on Formosa papaya: relatioships with soil water potencial, total soluble solids of latex and climate variables. Ciencia e Agrotecnologia, Lavras, v.32, n. 5, p. 1473-1480, 2008.

RODRÍGUEZ PASTOR, M. C. Consideraciones sobre la utilización de diferentes densidades en el cultivo de la papaya (Carica papaya, L.) "Baixinho de Santa Amalia" en las Islas Canarias. Revista Brasileira de Fruticultura, Jaboticabal, v. 24 n. 3, p.707-710, 2002.
RODRÍGUEZ PASTOR, M. C. Estudio sobre la fenología, comportamiento floral, productividad y características del fruto de papaya (Carica papaya, L.) en varios cultivares del grupo "Solo" con especial referencia a la carpeloidía en las condiciones canarias. 1989. $280 \mathrm{f}$. Tesis (Doctora en Biología) - Facultad de Biología, Universidad de La Laguna, Tenerife, 1989.

RODRÍGUEZ PASTOR, M. C.; GALÁN, V.; HERRERO, M. Evaluation of papaya autogamy. Fruits, Paris, v. 45, n. 4, p. 387-391, 1990

TECSON- MENDOZA, E. M. Development of functional foods in the Philipines. Food Science and Technology Research, Tsukuba, v.13, n. 3, p. 179-186, 2007. 